\title{
Qualification and implementation of line ratio spectroscopy on helium as plasma edge diagnostic at ASDEX Upgrade
}

\author{
M. Griener ${ }^{1,2}$, J.M. Muñoz Burgos ${ }^{3}$, M. Cavedon ${ }^{1}$, G. \\ Birkenmeier $^{2,1}$, R. Dux ${ }^{1}$, B. Kurzan ${ }^{1}$, O. Schmitz ${ }^{4}$, B. Sieglin ${ }^{1}$, \\ U. Stroth ${ }^{1,2}$, E. Viezzer ${ }^{5}$, E. Wolfrum ${ }^{1}$, the ASDEX Upgrade \\ Team $^{1}$ \\ ${ }^{1}$ Max Planck Institute for Plasma Physics, Boltzmannstr. 2, 85748 Garching, \\ Germany, \\ ${ }^{2}$ Physik Department E28, Technische Universität München, 85748 Garching, \\ Germany \\ 3 Astro Fusion Spectre, astrofusionspectre@gmail.com \\ ${ }^{4}$ Department of Engineering Physics, University of Wisconsin-Madison, USA \\ ${ }^{5}$ Dept. of Atomic, Molecular and Nuclear Physics, University of Seville, Avda. \\ Reina Mercedes, 41012 Seville, Spain. \\ E-mail: michael.griener@ipp.mpg.de
}

17 August 2017

\begin{abstract}
A new thermal helium beam diagnostic has been implemented as plasma edge diagnostic at the ASDEX Upgrade (AUG) tokamak. The helium beam is built to measure the electron density $n_{\mathrm{e}}$ and temperature $T_{\mathrm{e}}$ simultaneously with high spatial and temporal resolution in order to investigate steady-state as well as fast transport processes in the plasma edge region. For the thermal helium beam emission line ratio spectroscopy, neutral helium is locally injected into the plasma by a piezo valve. This enabled the measurement of the line resolved emission intensities of seven He I lines for different plasma scenarios in AUG. The different line ratios can be used together with a collisional-radiative model (CRM) to reconstruct the underlying electron temperature and density. Ratios from the same spin species are used for the electron density reconstruction, whereas spin mixed ratios are sensitive to electron temperature changes. The different line ratios as well as different CRMs are tested for their suitability for diagnostic applications. Furthermore their consistency in calculating identical parameters is validated and the resulting profiles are compared to other available diagnostics at AUG.
\end{abstract}

PACS numbers: 52

Keywords: fusion, plasma, scrape-off layer, ASDEX Upgrade, piezo valve, active spectroscopy, helium line ratio spectroscopy, collisional-radiative model, electron temperature, electron density 


\section{Introduction}

In devices for the magnetic confinement of fusion plasmas, such as tokamaks or stellarators, the plasma column outside the last closed flux surface (LCFS) features a rapid decay. In this region of open field lines, called scrape-off layer (SOL), heat and power are transported to the divertor via parallel transport or directly to the wall mainly due to convection by filaments. These mechanisms can seriously damage the first wall materials of the devices. Therefore, diagnostics for the SOL electron density $n_{\mathrm{e}}$ and electron temperature $T_{\mathrm{e}}$ with high spatial and temporal resolution are required to understand the filamentary structures as well as the power fluxes across the LCFS.

The active line ratio spectroscopy on He I emission lines offers these diagnostic capabilities. It enables the evaluation of the electron temperature and density simultaneously out of the measurement of at least three He I transitions.

While $n_{\mathrm{e}}$ measurements are also provided by the Li-beam diagnostic [1] as well as reflectometry [2], $T_{\mathrm{e}}$ is not readily available in this region of AUG. The electron cyclotron emission (ECE) diagnostic suffers from optical thin plasmas, the Thomson scattering (TS) diagnostic from the low signal levels and Langmuir probe measurements can only be done in weakly heated discharges.

The first idea of using helium line ratio spectroscopy for diagnostic purposes came up in the nineteen-fifties [3]. The main barriers for application were the poor knowledge of the excitation cross-sections needed for the collisional-radiative model (CRM) as well as low experimental light intensities. Big progress on the CRM had been bade by Brosda and co-authors [4], leading to the first application of a thermal helium beam in a fusion plasmas in the TEXTOR experiment in 1992 [5].

Helium line ratio spectroscopy can be applied on background helium as done in MST [6], NAGDIS-II [7], ASDEX Upgrade [8], heliac devices [9, 10] and the FT-2 tokamak [11]. For bigger devices, improved spatial resolution can be achieved by actively injecting helium into the plasma either by a supersonic beam [12] as done in TEXTOR [13] and TJ-IU [14]. An effusive as well as a thermal helium source had also been used at TEXTOR [13, 15]. Further, a thermal helium beam had been used in JET [16], the RFX reversed field pinch [17], RFX-mod [18, 19], W7-X [20] and is planned for WEST $[21]$.

The conventional approach is to use the singlet/singlet $(\mathrm{s} / \mathrm{s})$ ratio of $667.8 \mathrm{~nm}$ and $728.1 \mathrm{~nm}$ for density evaluation and the triplet/singlet $(\mathrm{t} / \mathrm{s})$ ratio of $706.5 \mathrm{~nm}$ and $728.1 \mathrm{~nm}$ for temperature determination $[5,15]$. The reason for this is that these lines with similar intensities are lying relatively close together which facilitates spectroscopic measurements.

Investigation of the temperature and density behaviour of He I lines has been done in linear plasma test stands [22, 23]. Although an extensive overview of possible helium lines was achieved, only a small range of plasma parameters, especially at lower densities, could be covered which made it difficult to predict the application possibilities for larger devices. 
In order to expand the line ratio technique to other helium line ratios, the absolute emission intensity of seven He I transitions were measured by a spectrometer in AUG. The different ratios were compared to select the ones with the best dependencies in a parameter range of $1 \times 10^{18} \mathrm{~m}^{-3}<n_{\mathrm{e}}<4 \times 10^{19} \mathrm{~m}^{-3}$ and $5 \mathrm{eV}<T_{\mathrm{e}}<250 \mathrm{eV}$, which are typical parameters for the SOL and pedestal region of AUG. The radially resolved emission information is used to validate a new CRM for He [24, 25].

This paper describes the implementation of a thermal helium beam [26] as plasma edge diagnostic for ASDEX Upgrade (AUG) as well as the results gained during the first experimental campaign: The physical basics of the helium line ratio spectroscopy are explained in section 2 and the implementation of the new thermal helium beam diagnostic in the ASDEX Upgrade experiment is described in section 3. In section 4 the experimental results are covered. In section 5 multiple line ratios are investigated in order to identify suitable combinations showing a strong dependence on electron temperature and density changes. The new CRM is used to discriminate the effect of $T_{\mathrm{e}}$ and $n_{\mathrm{e}}$ on the behaviour of the line ratios.

The achieved results with different atomic data bases are compared in section 6 and bench-marked with well-established diagnostics. Finally, in section 7 , the first results of the thermal helium beam at AUG are summarised which lead to hardware enhancements in order to gain spatially and temporally highly resolved $n_{\mathrm{e}}(r, t)$ and $T_{\mathrm{e}}(r, t)$ profiles in the SOL of AUG for the upcoming campaigns.

\section{Diagnostic principle of helium line ratio spectroscopy}

For neutral helium the electronic system is divided into singlet and triplet states as shown in the Grotrian diagram of He in Figure 1. The simplicity of this two electron system, which is easier to describe compared to other noble gases where the optical transitions are also split into singlet and triplet states, is one advantage of helium as spectroscopy gas. This led to further improvements in the CRM for helium [25] which is crucial for data evaluation.

The principle of the helium line ratio spectroscopy is based on the different behaviour of the singlet and triplet state population density on changes of $n_{\mathrm{e}}$ and $T_{\mathrm{e}}$. While the triplet state population density peaks at a temperature of $T_{\mathrm{e}} \approx 25 \mathrm{eV}$, the singlet states have their maximum of the population density at $T_{\mathrm{e}} \approx 300-500 \mathrm{eV}$ $[13,27]$. Therefore, for high temperatures, the population density of singlet states is increased relative to triplet states.

The density dependence can by explained by the different type of the dominant depopulation processes. The population of states with the main quantum number $n \geq 3$ is dominated by electron collisions $\left(\sim n_{\mathrm{e}}\right)$, whereas the excited state is either dominantly depopulated by radiation (independent of $n_{\mathrm{e}}$ ) or by electron collisions $\left(\sim n_{\mathrm{e}}\right)$. Consequently, the line ratio of such two states is electron density sensitive.

In general this means that the line intensity ratios of two suitable singlet or triplet lines combined with a collisional radiative model (CRM) enable the reconstruction of the 
underlying values for the electron density, whereas singlet/triplet ratios yield $T_{\mathrm{e}}$ values. The optical transitions of neutral helium in the visible range are listed in Table 1. The measured relative emission intensities strongly depend on the plasma density and temperature as this is the function principle of the diagnostic. The measured intensities including the error interval which takes these changes into account are compared to the intensity data from from NIST [28].

All lines are plotted in Figure 1, where the line thickness represents their relative intensity. Beside the dashed lines, all these wavelengths had been measured in the AUG by spectroscopy on helium which is locally injected in the deuterium plasma discharges as explained in the following section.

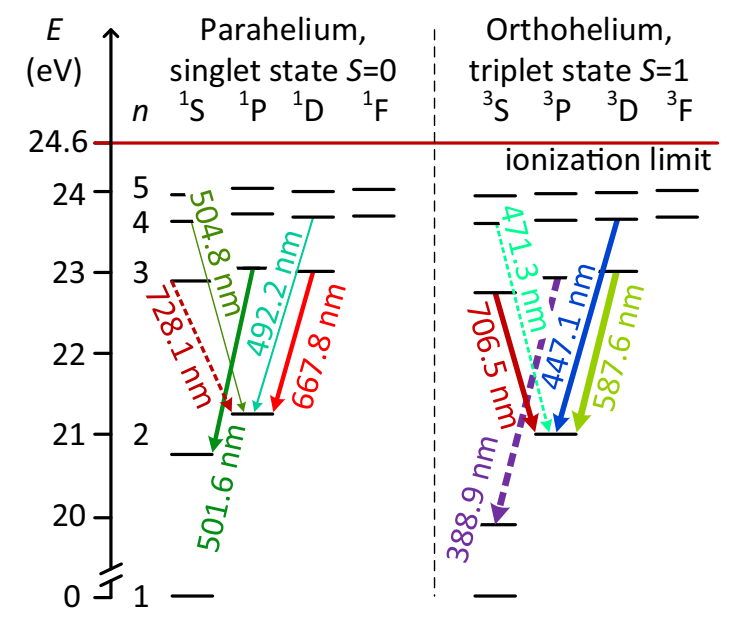

Figure 1: He I Grotrian diagram, divided into singlet and triplet states. All lines are listed in Table 1. The solid drawn lines had been measured within this work. 
Table 1: Optical transitions of He I in the visible spectral range, plotted in Figure 1. The relative intensities from NIST [28] are compared to the mean measured intensities where the standard deviation denotes the change of the emission for different plasma parameters.

\begin{tabular}{c|c|c|c|c}
\hline $\begin{array}{c}\text { wavelength } \\
(\mathrm{nm})\end{array}$ & species & transition & $\begin{array}{c}\text { rel. int. } \\
\text { (NIST) }\end{array}$ & $\begin{array}{c}\text { mean rel. int. } \\
\text { (measured) }\end{array}$ \\
\hline 388.86 & triplet & 1s3p - 1s2s & 500 & - \\
447.15 & triplet & 1s4d - 1s2p & 200 & $200 \pm 100$ \\
471.31 & triplet & 1s4s - 1s2p & 30 & - \\
492.19 & singlet & 1s4d - 1s2p & 20 & $70 \pm 30$ \\
501.56 & singlet & 1s3p - 1s2s & 100 & $100 \pm 40$ \\
504.77 & singlet & 1s4s - 1s2p & 10 & $10 \pm 4$ \\
587.56 & triplet & 1s3d - 1s2p & 500 & $2800 \pm 1600$ \\
667.81 & singlet & 1s3d - 1s2p & 100 & $620 \pm 250$ \\
706.52 & triplet & 1s3s - 1s2p & 200 & $590 \pm 360$ \\
728.13 & singlet & 1s3s - 1s2p & 50 & - \\
\hline
\end{tabular}

\section{Experimental setup}

Beside measuring helium line ratios in plasmas with an intrinsic helium content [29] it is possible to locally inject helium into plasmas to perform active spectroscopy as done in AUG. The first advantage of helium as spectroscopy gas is its well described electronic system. The second advantage lies in the ionisation energy of $24.6 \mathrm{eV}$, which is the highest first ionisation energy of all atoms. Therefore, neutral helium atoms injected as a thermal or supersonic beam can penetrate relatively deeply into the plasma.

In AUG a piezo valve based system for the injection of thermal gas beams [26] is used to locally inject helium into the plasma SOL. The compact valve is placed at the low field side at the midplane of the vacuum chamber at a major radius of $R=2.23 \mathrm{~m}$. The gas expands through a thin capillary and expands in a cone shaped gas beam with a full opening angle of about $40^{\circ}$ for helium. Regulated by the filling pressure of the valve, gas fluxes are accessible from $10^{18}$ up to $10^{23}$ part/s. The gas flux can be modulated with switch on/off times of $\leq 0.5 \mathrm{~ms}$.

The position of the valve relative to the plasma and the lines of sight (LOS) is represented in a toroidal cross section of AUG in Figure 2. The endpoint of the capillary for gas inlet lies protected by a tungsten coated carbon brick $2 \mathrm{~cm}$ behind the limiter contour. The central axis of the gas beam is directed perpendicular to the magnetic flux surfaces. 21 LOS from three optical heads (pictured with three different shades of blue), which had been optimised for charge-exchange measurements at AUG [30, 31], are almost tangential to the field lines in the measurement area over the radial range of $4 \mathrm{~cm}$.

To map the measured line integrated intensity of each LOS to a radial position, a forward 


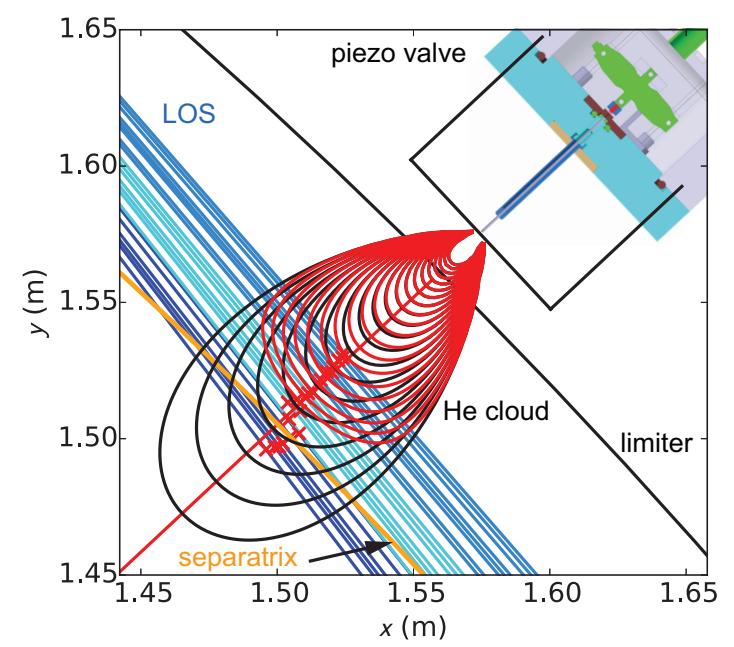

Figure 2: Toroidal cross section of AUG at $z=0.05 \mathrm{~m}$. The LOS, the piezo valve and a contour plot of the helium density (black - without ionisation, red - with ionisation) as well as the separatrix (orange, $R=2.12 \mathrm{~m}$ ) and limiter position are illustrated.

model calculates the spatial origin of the helium emission along each LOS. The helium beam expansion is modelled based on measurements of the undisturbed gas cloud shape with a fast camera [26]. In addition to this undisturbed shape which is presented in Figure 2 by black contour lines, one has to take ionisation effects into account. This becomes mainly important in the confined region and the near SOL as there the neutral helium density is attenuated strongly. The calculated shape of the cloud including ionisation processes in the AUG plasma is presented by red contour lines, representing the same density values as the black lines.

The radii of maximal emission, marked in Figure 2 by red crosses, and the radial intervals which cover $68 \%$ of the emission along a LOS are set as radial origin and its error of the signal, respectively. The radial resolution ranges from $3 \mathrm{~mm}$ (outer most LOS, closest to the gas injection point) to $7 \mathrm{~mm}$ (innermost LOS).

For the analysis of the helium emission, the collected light is transmitted from the optical heads by $400 \mu \mathrm{m}$ optical fibers $(\mathrm{NA}=0.22)$ to a high through-put $f / 4$ Czerny-Turner spectrometer with a variable spectrometer slit of typically $50 \mu \mathrm{m}$, two objective lenses and a turnable grating with 2400 grooves $/ \mathrm{mm}$. The imaging is based on a Princeton Instruments "ProEM" charge coupled device (CCD) camera with a $512 \times 51216 \mu \mathrm{m}$ pixel chip. This limits the temporal resolution to $2.3 \mathrm{~ms}$ (cf. [30, 32]). The covered wavelength range for one spectrometer setting lies between $14 \mathrm{~nm}$ for the lowest and $7 \mathrm{~nm}$ for the highest measured wavelength.

This spectrometer did not cover the highest $(728.1 \mathrm{~nm})$ and lowest $(388.9 \mathrm{~nm})$ wavelengths of the discussed He I lines which is the reason for the lack of experimental data here. The $447.3 \mathrm{~nm}$ line was skipped due to the limited number of available plasma discharges. 


\section{Measurement results}

\subsection{Experimental sequence}

In the AUG campaign 2015, six comparable discharges (\#32 029 - \#32 032, \#32 046 , \#32 047) had been performed to measure the intensity profiles of seven He I transitions. Due to the high resolution of the spectrometers (cf. subsection 4.2), one helium line was measured per discharge, beside the 501.56 and $504.77 \mathrm{~nm}$ lines which are close enough to be measured within one discharge at the same spectrometer setting.

One of the identical discharges is presented in Figure 3. They are designed in a way to cover a wider range of typical SOL parameters between $1 \times 10^{18} \mathrm{~m}^{-3} \leq n_{\mathrm{e}} \leq$ $4 \times 10^{19} \mathrm{~m}^{-3}$ and $5 \mathrm{eV} \leq T_{\mathrm{e}} \leq 130 \mathrm{eV}$. This is enabled by a step wise increase of the electron cyclotron resonance heating power $P_{\mathrm{ECRH}}$, the neutral beam heating power $P_{\mathrm{NBI}}$ (see Figure $3 \mathrm{~b}$ )) and the plasma fuelling (Figure $3 \mathrm{c}$ ) and d)) during the discharge. The discharges start in the low confinement mode (L-mode) with a transition to the high confinement mode (H-mode) at $\sim 3 \mathrm{~s}$, which is accompanied by the appearance of edge localised modes (ELMs). ELMs are identified by peaks in the divertor shunt current signal $I_{\text {i-DIV }}$ (Figure 3 e)).

The position of the separatrix at the midplane is given by the parameter $R_{\text {aus }}$ (Figure 3 f)). This position is periodically shifted five times (marked by the vertical black lines) to cover different regions in the plasma with the diagnostics which are spatially fixed such as the Li- or He-beam or the Thomson scattering diagnostic.

For the helium beam measurements, the piezo valve with a $700 \mu \mathrm{m}$ capillary was operated with 50 mbar helium. This leads to a gas flux of $1.68 \times 10^{19} \mathrm{part} / \mathrm{s}$, which is around 600 times less than the continuously injected deuterium for plasma fuelling (Figure $3 \mathrm{~d}$ )). The helium beam was pulsed with $10 \mathrm{~Hz}$, leading to puffs with $50 \mathrm{~ms}$ duration, where the downtime was used for background subtraction. The emission time trace of the helium $667.8 \mathrm{~nm}$ line is shown Figure $3 \mathrm{~g}$ ) with two signals from two different radial positions, dedicated to the inner an outermost LOS as shown in Figure 2. The emission behaviour is discussed in subsection 4.3 .

\subsection{Spectral profiles of He I transitions}

The experimental equipment used offers a wavelength resolved emission profile for each line of sight. Although this is not crucial for the helium line ratio technique, the detailed analysis of the spectral lines helps to identify other emission lines which may disturb the helium measurements. Elements which are often found in a nuclear fusion device are hydrogen and its isotopes as main plasma species, carbon and tungsten as plasma wall materials, boron for wall conditioning, nitrogen and oxygen as impurities from air, or as nitrogen, neon and argon injected on purpose for seeding experiments or as diagnostic gases [20].

In the experiments performed, all He I lines could be clearly resolved from other transitions in the measurement region. The exact wavelengths and measurement ranges 


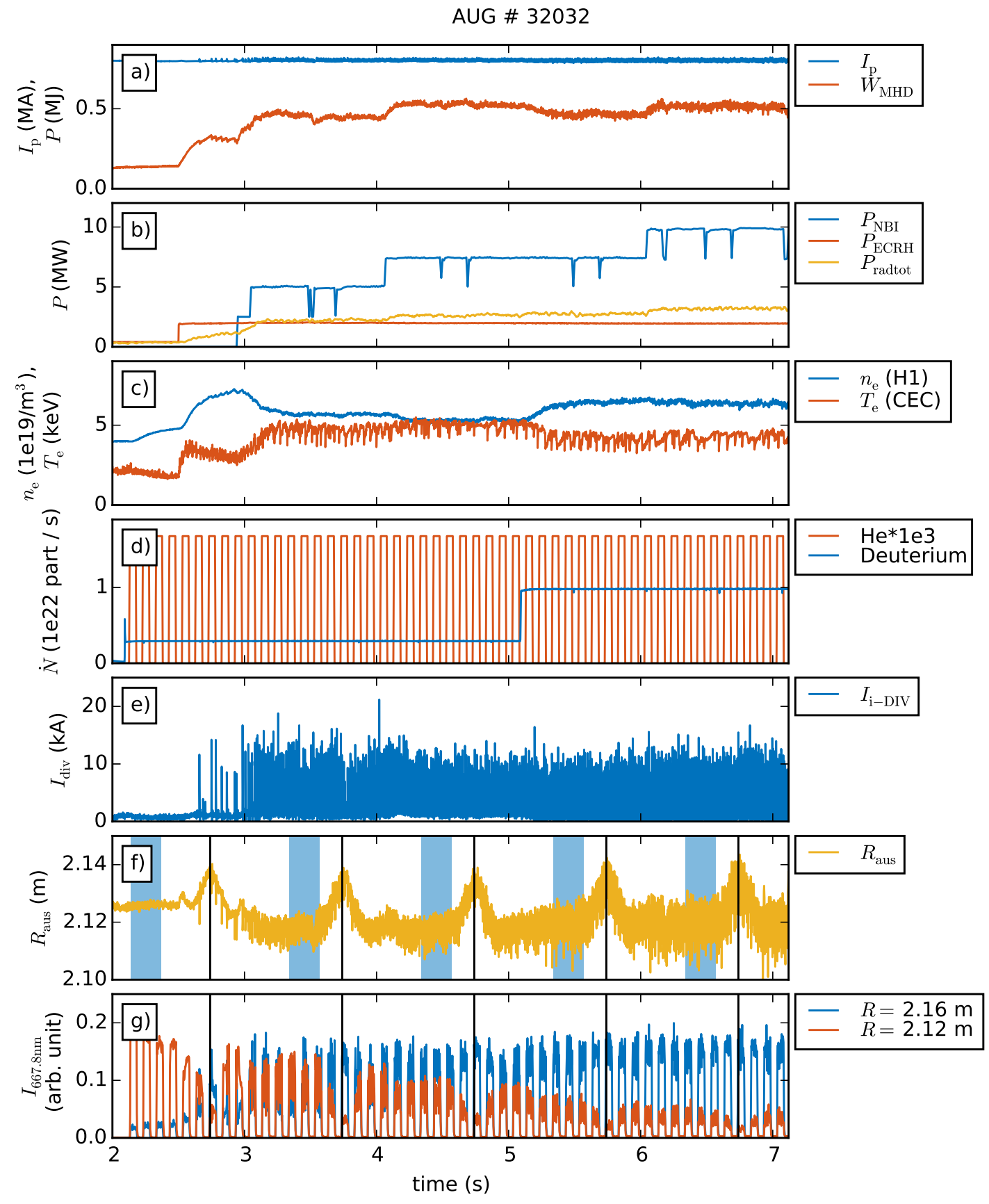

Figure 3: Experimental timetraces of AUG discharge \#32 032. The timetraces show the plasma current $I_{\mathrm{P}}$, the total stored energy in the plasma $W_{\mathrm{MHD}}$, the neutral beam injection and electron cyclotron resonance heating power $P_{\mathrm{NBI}}$ and $P_{\mathrm{ECRH}}$, the total radiated power $P_{\text {radtot }}$, the line integrated electron density $n_{\mathrm{e}}$ and core electron temperature $T_{\mathrm{e}}$, the total plasma fuelling with deuterium compared to the diagnostic He inlet, the shunt current of the inner divertor $I_{\mathrm{i}-\mathrm{DIV}}$, the separatrix position at the midplane $R_{\text {aus }}$ and the measured emission $I_{667.8 \mathrm{~nm}}$ at $667.8 \mathrm{~nm}$ of the injected helium at two radial positions (cf. subsection 4.3). 
are summarised in Table 2 and Figure 4 . Beside the very strong $H_{\beta}$ line (as deuterium was used, it is actually the $D_{\beta}$ line), all additional peaks found are weaker than the helium lines and can be allocated to W I emission as ASDEX Upgrade is a equipped with a full tungsten wall.

Table 2: Emission lines close to He I transitions. Beside the very strong $H_{\beta}$ line, all peaks found in the measurement range can be allocated to W I emission. The presence of neon (Ne I-II) would mostly interfere with the He I lines.

\begin{tabular}{|c|c|c|c|c|c|}
\hline transition & $\begin{array}{l}\text { wavelength } \\
\text { (nm) }\end{array}$ & $\begin{array}{l}\text { discharge } \\
\text { number }\end{array}$ & $\begin{array}{l}\text { measurement } \\
\text { range }(\mathrm{nm})\end{array}$ & perturbation lines measured (nm) & $\begin{array}{c}\text { closest } \mathrm{Ne} \\
\text { line }[28]\end{array}$ \\
\hline $3^{3} \mathrm{P} \rightarrow 2^{3} \mathrm{~S}$ & 388.86 & - & - & - & 388.94 \\
\hline $4^{3} \mathrm{D} \rightarrow 2^{3} \mathrm{P}$ & 447.15 & 32046 & $703-710$ & $\begin{array}{c}441.4,441.6,446.4,448.6 \\
449.7,451.0,451.4\end{array}$ & 447.15 \\
\hline $4^{3} \mathrm{~S} \rightarrow 2^{3} \mathrm{P}$ & 471.31 & - & - & - & 471.30 \\
\hline $4^{1} \mathrm{D} \rightarrow 2^{1} \mathrm{P}$ & 492.19 & 32047 & $485-498$ & $486.1\left(H_{\beta}\right), 491.8,494.1,494.5$ & 492.82 \\
\hline $3^{1} \mathrm{~S} \rightarrow 2^{1} \mathrm{~S}$ & 501.56 & 32030 & 496-509 & $500.1,500.5$ & 501.52 \\
\hline $4^{1} \mathrm{~S} \rightarrow 2^{1} \mathrm{P}$ & 504.77 & 32030 & $496-509$ & 504.5 & 504.66 \\
\hline $3^{3} \mathrm{D} \rightarrow 2^{3} \mathrm{P}$ & 587.56 & 32031 & $582-594$ & none & 587.28 \\
\hline $3^{1} \mathrm{D} \rightarrow 2^{1} \mathrm{P}$ & 667.81 & 32032 & $663-671$ & none & 667.82 \\
\hline $3^{3} \mathrm{~S} \rightarrow 2^{3} \mathrm{P}$ & 706.52 & 32029 & $703-710$ & none & 706.47 \\
\hline $3^{1} \mathrm{~S} \rightarrow 2^{1} \mathrm{P}$ & 728.13 & - & - & - & 724.52 \\
\hline
\end{tabular}

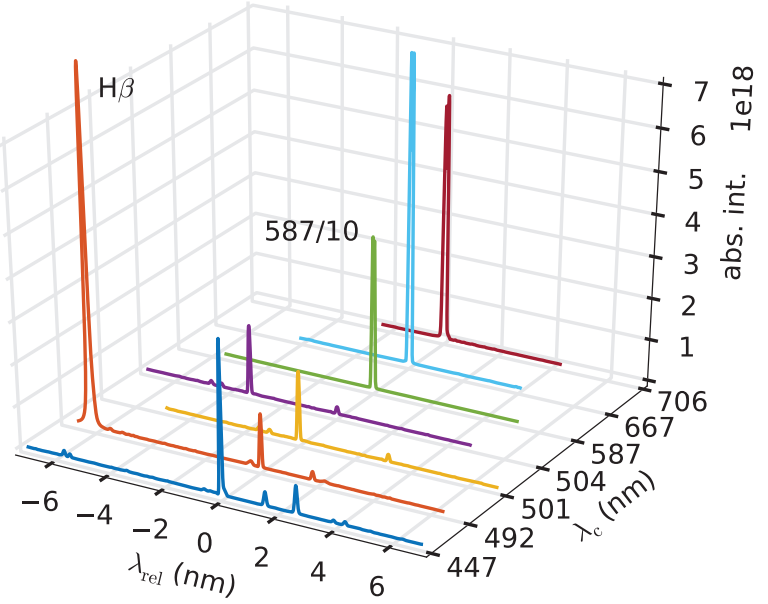

Figure 4: Measured spectra $\lambda_{\text {rel }}$ of He I lines where the central wavelength $\lambda_{\mathrm{c}}$ of the transitions correspond to $\lambda_{\text {rel }}=0$. The data are a time averaged mean of all LOS during the He puffs. The strongest signal which corresponds to the $587 \mathrm{~nm}$ line is divided by ten.

The presence of nitrogen would not disturb the measurements. If a filter based system 
instead of spectrometers is used for the measurements, it is of particular interest that all nitrogen lines for $\lambda \geq 587 \mathrm{~nm}$ are separated more than $1.1 \mathrm{~nm}$ from the He I lines, respectively.

In contrast to nitrogen, the presence of noble gases as neon and argon would mostly interfere with the exclusive measurement of He I lines. The highest distance (in brackets) of He I lines to Ne lines show the $728.1 \mathrm{~nm}(3.6 \mathrm{~nm}), 492 \mathrm{~nm}(0.6 \mathrm{~nm}), 587 \mathrm{~nm}(0.3 \mathrm{~nm})$, $504 \mathrm{~nm}(0.1 \mathrm{~nm})$ and $388 \mathrm{~nm}(0.08 \mathrm{~nm})$ lines. These could still be resolved with a high resolution spectrometer. Nevertheless the Zeeman splitting of the lines have to be taken into account so that some of these lines cannot be distinguished anymore.

The Zeeman splitting for example splits the $3^{1} \mathrm{D} \rightarrow 2^{1} \mathrm{P}$ transition into 9 allowed transitions where the $\sigma^{+}$and $\sigma^{-}$lines are separated by $0.08 \mathrm{~nm}$ at a magnetic field of $2 \mathrm{~T}$ at the measurement position (cf. Figure 5). The angle between the magnetic field and the observation direction was around $5^{\circ}$ which let the $\pi$ lines nearly vanish.

Figure 5 shows that the measured spectral profile fits the predictions by a forward modelling taking the spectrometer function and the Zeeman splitting into account.

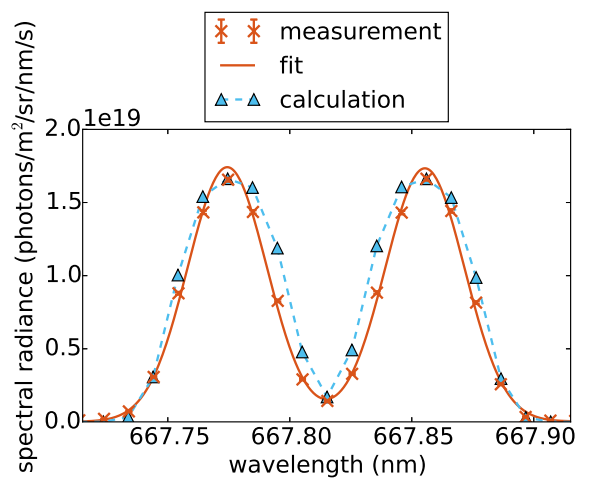

Figure 5: Spectrum of the He I 667.8 nm line, measured in the AUG discharge \#32 167 with the outermost LOS. Compared to a forward modelling taking the spectrometer function and the Zeeman splitting into account.

Comparing the line intensities before the first, during and after the last helium puff, as done in Figure 6, shows on the one hand the very good signal-to-noise ratio during helium puffs and on the other hand the fast depletion of neutral helium in the SOL. This had also been confirmed in [26], by showing that the influence of the helium puffing onto the global helium content in the plasma core lies in the order of $0.1 \%$, which does not affect the plasma performance.

As detailed analysis of the inner divertor shunt current $I_{\mathrm{i}-\mathrm{DIV}}$ (cf. Figure 3 for an overview timetrace) shows that the helium puffs had no influence on the ELM behaviour. As ELMs react very sensitively to perturbations of the plasma edge region this is an additional indicator that the operation of the piezo valve with low helium fluxes causes neglegible disturbance.

In the subsequent analysis only the total line emission intensities are of interest and the integral value of the helium peaks was taken. This region of interest is shown as the 
highlighted region in Figure 6 for the $667.8 \mathrm{~nm}$ line.

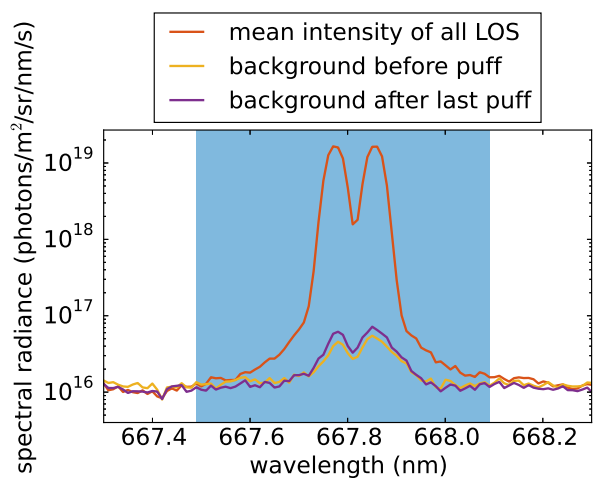

Figure 6: Spectrum of the He I $667.8 \mathrm{~nm}$ line in logarithmic scale as a mean of all LOS (AUG \#32 167), comparing the signal before, during and directly after He puffs.

\subsection{Helium emission intensity time traces from L- to H-mode}

The intensity time trace of the examplarily chosen He I singlet transition at $667.8 \mathrm{~nm}$ is shown in Figure $3 \mathrm{~g}$ ) for two different radial positions. The first is the separatrix position at $R=2.12 \mathrm{~m}$ (at the height $z=0.05 \mathrm{~m}$, where the valve is located), the second is lying in the far scrape-off layer at $R=2.16 \mathrm{~m}$, with $6 \mathrm{~cm}$ distance to the helium injection point. The fifty $50 \mathrm{~ms}$ helium puffs with a duty cycle of $100 \mathrm{~ms}$ are clearly visible for all measurement locations.

Note that the emission intensity in L-mode (at the beginning of the discharge) is highest in the confined region of the plasma $(R \leq 2.125 \mathrm{~m})$, although the neutral helium density is higher in the far SOL. The reason for this is that the excitation of the neutral helium is much more efficient at the temperatures in the separatrix region, compared to that at the low temperatures in the far SOL.

In $\mathrm{H}$-mode this effect is compensated by increasing SOL temperatures (probably due to warm filaments, which cannot be resolved in the present setup) on the one hand, which lead to an increased emission at $R=2.16 \mathrm{~m}$, and by the increasing amount of ionisation of the injected helium in the confined region on the other hand, which decreases the number of neutral helium atoms.

As temperature and density are increased step wise during the discharge, the ionisation losses gain more and more importance, as the emission drop in the separatrix region shows. The high ionisation in the confined region is pronounced by the $R_{\text {aus }}$ scans which are indicated by the five vertical lines in the subplots f) and g) of Figure 3. At these times, the position of the separatrix is shifted closer to the plasma wall so that the measurement points are shifted more towards the plasma centre, where the density of neutral helium is strongly decreased by ionisation and thus the total emission intensity is decreased, too.

During H-mode, the $R_{\text {aus }}$ scans do not influence the emission intensity in the far SOL. 
The reason for this is that the electron density and temperature profiles (cf. Section 6) do not show steep gradients in this region. Furthermore, ionisation does not influence the neutral density strongly in the far SOL as shown in Figure 2.

Regarding the emission time traces, it is clear that the intensity variation within the single puffs is strongly increased in H-mode with respect to L-mode. This emission variation is caused by ELMs which directly influence the electron temperature and density in the measurement region and thus lead to a changed helium emission. The time resolution of $2.3 \mathrm{~ms}$ is not sufficient to resolve single ELMs. To achieve comparable results, only measurement points in between ELMs were taken into account for the time averaged signals which are used for the radial profiles.

\subsection{Radial profiles of He I transitions in L- and H-mode}

The information from each line of sight can be mapped to a radial position as shown in section 3. Taking all LOS together leads to radial profiles as shown in Figure 7 for the L-mode phase and in Figure 8 for an H-mode phase with high deuterium fuelling and high heating power. The intensity values of all optical transitions are time averaged over the ELM free points in the denoted time spans with constant plasma parameters. The separatrix position $\left(\rho_{\text {pol }}=1.0\right)$ is given by the vertical black lines. This line divides the confined region (to the left of the line) and the SOL (to the right of the line) of the plasma. The helium is injected from right to left starting at a radius of $R=2.23 \mathrm{~m}$.

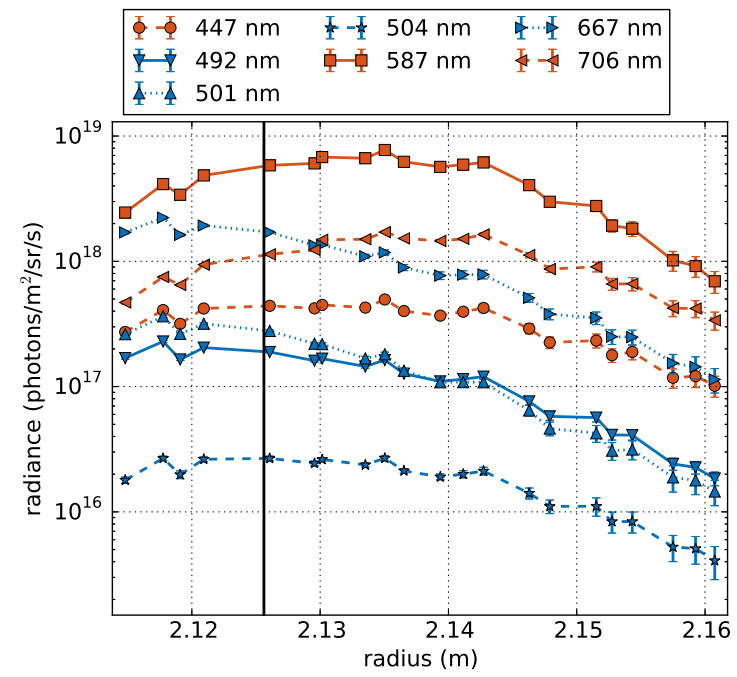

Figure 7: Radial profile of the absolute emission intensity of He I transition in logarithmic scale (blue - singlet, red - triplet) for an AUG L-mode discharge (\#32 029 32 032, \#32046 and \#32 047, $t=2.17-2.37 \mathrm{~s})$.

Also the neutral helium density decreases from right to left due to geometrical dilution and ionisation losses, the total emission intensity in L-mode increases towards the plasma center. This is explained with the more efficient excitation at higher temperatures. In 


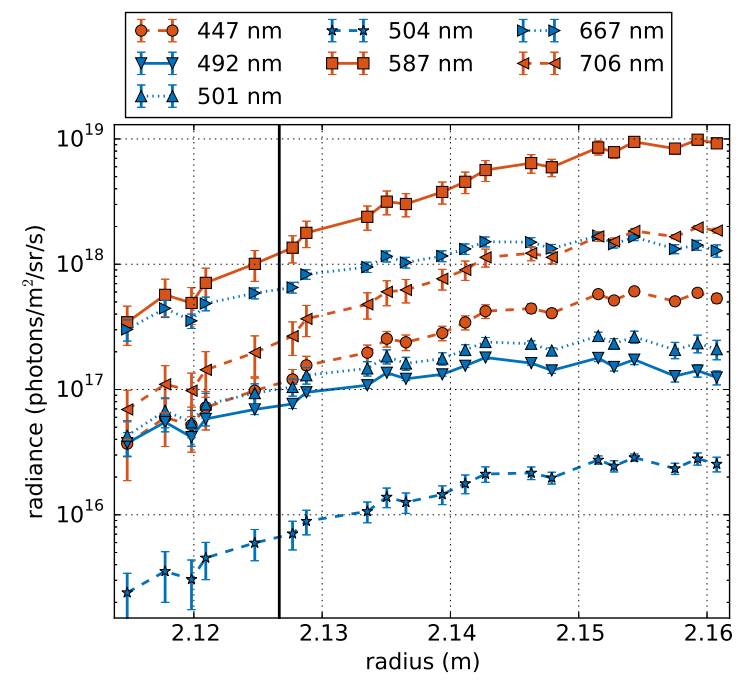

Figure 8: Radial profile of the absolute emission intensity of He I transition in logarithmic scale (blue - singlet, red - triplet) for an AUG H-mode discharge (\#32 029 32 032, \#32046 and \#32 047, $t=6.34-6.57 \mathrm{~s})$.

H-mode, however, beam attenuation via ionisation dominates towards the center leading to a decreased total emission, as discussed earlier.

In L-mode the singlet line emission profiles (blue) are mainly increasing continuously towards the plasma center whereas the triplet lines (red) are peaking already in the SOL region. The reason for this difference is the different temperature behaviour of the population density of singlet and triplet lines as explained in section 2 . The population density of singlet lines peaks at $T_{\mathrm{e}} \approx 300-500 \mathrm{eV}$ which is far in the confined region, whereas the population density of triplet lines peaks at $T_{\mathrm{e}} \approx 25 \mathrm{eV}$ which lies in the SOL [13]. This also causes the steeper decrease in the H-mode of triplet line emission compared to singlet line emission. By taking the intensity ratio of two lines the absolute intensity, which is proportional to the neutral helium density, cancels out. The line ratio thus only reflects the changes of $T_{\mathrm{e}}$ and $n_{\mathrm{e}}$.

\section{Feasible He I line ratios for $n_{\mathrm{e}}$ and $T_{\mathrm{e}}$ measurements}

From four measured He I singlet line intensities and three measured triplet intensities one can calculate 21 different ratios, distributed into 12 singlet/triplet, 6 singlet/singlet and 3 triplet/triplet ratios.

These ratios were tested regarding their suitability for $n_{\mathrm{e}}$ and $T_{\mathrm{e}}$ evaluation for different plasma scenarios in AUG. These scenarios were realised in repeated AUG discharges (cf. Figure 3) by a step wise increase of plasma heating and plasma fuelling. Five time periods with constant plasma parameters in each discharge were investigated.

As the temperature as well as the density of the plasma strongly decay in the scrape-off layer, the measured ratios should show a strong dependence on the plasma radius (cf. 
Figure 14). This is a necessary criterion for the usability of the given ratio as $n_{\mathrm{e}}$ or $T_{\mathrm{e}}$ diagnostic. Furthermore a high intensity of both transitions of the ratio would be preferable to enable fast measurements.

To rate the ratios according to these criteria, their relative variation in the investigated radial range, i.e. $\max / \mathrm{min}$, is compared. This value, together with the absolute maximum and minimum values of all ratios along the radius are given in Table A1 for the L-mode and first H-mode timespan. The given relative intensity is averaged over the radius and corresponds to the intensity of the weaker line of the ratio with respect to the weakest intensity of all lines for all measured plasma scenarios.

Furthermore, the relative variation of all ratios is plotted in the left subfigure of Figure 9 for all singlet/triplet (s/t) ratios and in the right subfigure for $\mathrm{s} / \mathrm{s}$ and $\mathrm{t} / \mathrm{t}$ ratios for five different plasma scenarios. The ratios are ordered by the intensity of the weaker line of the ratio.
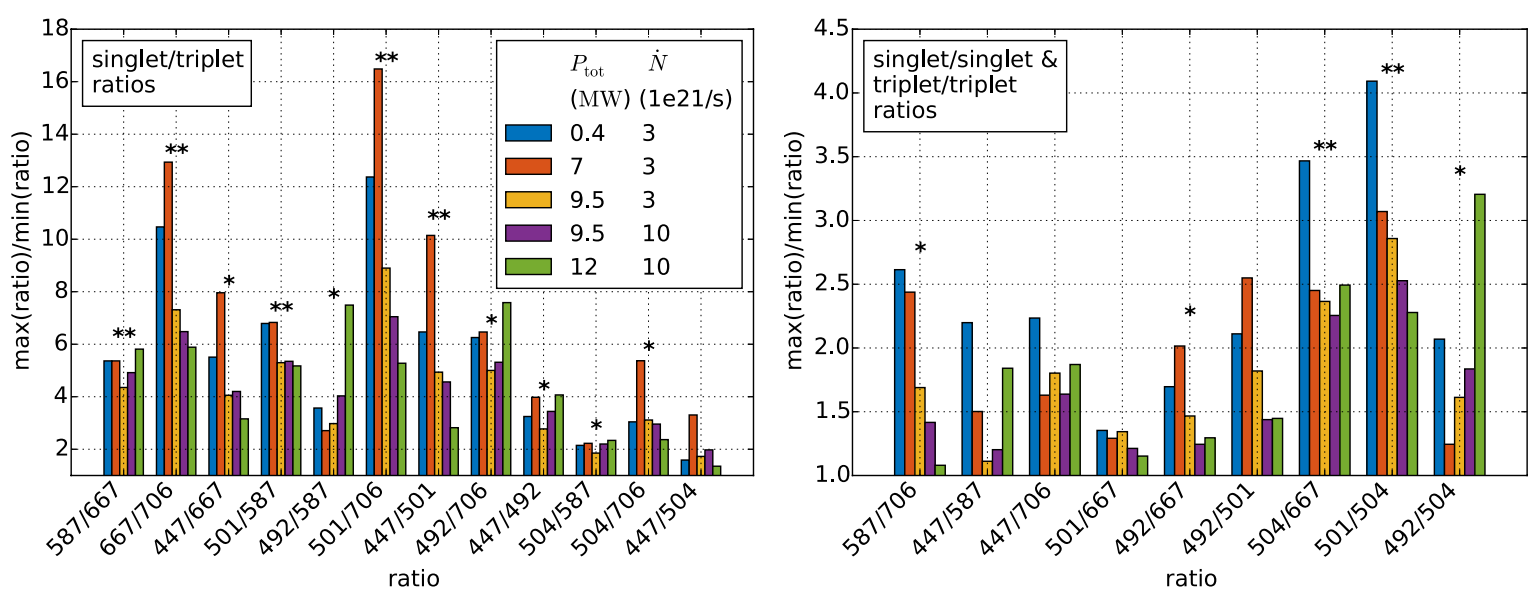

Figure 9: Comparison of the radial dependence of $T_{\mathrm{e}}$ sensitive line ratios (left) and $n_{\mathrm{e}}$ sensitive line ratios (right) for five dedicated time periods during the AUG discharge (cf. Figure 3). The legend shows the total heating power $P_{\text {tot }}$ as well as the plasma fuelling $\dot{N}$ with deuterium. The ratios are ordered by decreasing intensity of the weaker line of the ratio. Two asterisks denote the usability of the ratio for $n_{\mathrm{e}}$ or $T_{\mathrm{e}}$ evaluation, one asterisk denotes a general segregated $n_{\mathrm{e}}$ or $T_{\mathrm{e}}$ dependence.

In general the values of $\mathrm{s} / \mathrm{t}$ ratios show a stronger change along the radius compared to $\mathrm{s} / \mathrm{s}$ or $\mathrm{t} / \mathrm{t}$ ratios. Furthermore, the change of the ratio along the radius is highest for the first $\mathrm{H}$-mode timespan for most of the $T_{\mathrm{e}}$ dependent ratios (cf. Figure 9, left). The reason is that the temperature decrease around the separatrix is higher in H-mode compared to L-mode, which leads to a higher temperature range. With increasing plasma heating and fuelling this effect is compensated as the temperatures are still high in the far SOL (cf. [33]), which leads to a smaller variation of the ratios.

Beside the radial dependence of the ratio, an additional criterion for application as diagnostic is that the ratio depends on changes in $T_{\mathrm{e}}$ or in $n_{\mathrm{e}}$ but not in both simultaneously. Moreover, the translation into $T_{\mathrm{e}}$ and $n_{\mathrm{e}}$ values is only possible, if 
the measured ratio can be predicted with the CRM.

The fulfilment of these two criteria is denoted with asterisks in Figure 9. The ratio is rated with one asterisk if the $T_{\mathrm{e}}$ and $n_{\mathrm{e}}$ dependencies are clearly separated in the CRM and with two asterisks if in addition the absolute value of the measured ratio matches roughly the predictions of the CRM.

The traditionally used density dependent ratio is the 667/728 (s/s) ratio [15]. Figure 9 (right) shows that especially the $504.8 \mathrm{~nm}$ transition is well suited to replace the $728.1 \mathrm{~nm}$ line. Both transitions change the total orbital momentum by $\Delta L=+1$ and both corresponding upper states are mainly depopulated by electron collisions. In contrast to that, the upper states of singlet transitions with $\Delta L=-1$ are mainly depopulated by radiation [13]. So it can be concluded that $\mathrm{s} / \mathrm{s}$ ratios are best suitable for density evaluation if one transition shows $\Delta L=+1$ and the other $\Delta L=-1$. However also the $4^{1} \mathrm{D}$ state, which is the upper state of the $492.2 \mathrm{~nm}$ line, is partially depopulated by electron collisions. This could enable this transition to be also used for $n_{\mathrm{e}}$ reconstruction in combination with the $667.8 \mathrm{~nm}$ line.

As an example for an $n_{\mathrm{e}}$ dependent ratio including the $504.8 \mathrm{~nm}$ line, the $T_{\mathrm{e}}$ and $n_{\mathrm{e}}$ dependent value of the $501 / 504(\mathrm{~s} / \mathrm{s})$ ratio is plotted in Figure 10 for three different CRMs. The left subplot shows the predictions of the CRM included in ADAS using the 'pec96\#he_pju\#he0.dat' data set [34]. Here the single excited levels are treated as individual up to $n=4$. Before the ionisation state which is treated as loss term, one bundled state is added. This state gets populated by collisions from lower states and itself can populate lower states again, described by the so called bundle-n approach [35].
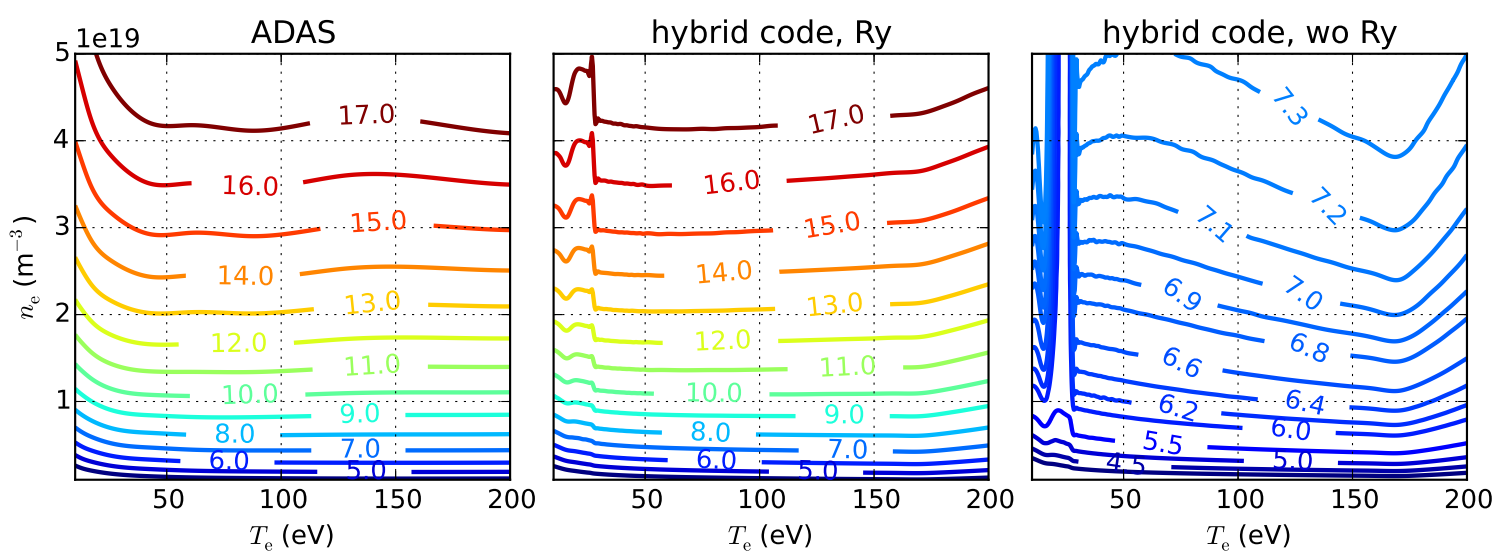

Figure 10: Comparison of the $T_{\mathrm{e}}$ and $n_{\mathrm{e}}$ behaviour of the 501/504 (s/s) ratio for three different CRMs. The left is the CRM implemented in ADAS [34], the middle is the hybrid code from [25] with high Rydberg states included, the right is the same code from [25], but without the high Rydberg states. The absolute number of these predicted values for the ratio can be compared to the experimental values in Table A1 and Figure 14.

The predicted values of the ADAS CRM are in agreement with the predictions done 
by the hybrid code by Muñoz Burgos et al. [25], which includes high Rydberg (Ry) states up to $n=500$, although this high number of states is physically not possible. The spikes of the line ratios at low temperature can be explained by the resonances of the electron-impact excitation cross-sections calculated using both the R-Matrix, and R-Matrix with Pseudo-States (RMPS) methods [36].

The same hybrid code without high Rydberg states included stops at $n=4$ and directly considers every higher excitation as ionisation. The predictions without high Ry states deliver significantly lower numbers of the ratio, which means that the population of the $4^{1} \mathrm{~S}$ state is overestimated with respect to the $3^{1} \mathrm{P}$ state. Therefore an $n_{\mathrm{e}}$ reconstruction is not possible without the high Ry states as the measured values of the ratio up to 16 would lead to unreasonable high densities.

Nevertheless, the ADAS model as well as the hybrid code with Ry lead to well suitable density profiles if the $504.8 \mathrm{~nm}$ lines is used, as shown in section 6 . The disadvantage of the $504.8 \mathrm{~nm}$ line is that it is the weakest of all He I lines and therefore not suitable for fast measurements. For a time resolution of $2.3 \mathrm{~ms}$ however, the absolute emission intensity was sufficient for all measured helium lines.

Beside $\mathrm{s} / \mathrm{s}$ ratios, $\mathrm{t} / \mathrm{t}$ ratios should also show a distinct $n_{\mathrm{e}}$ dependence. The 587/706 $(\mathrm{t} / \mathrm{t})$ ratio is the triplet counterpart to the classically used $\mathrm{s} / \mathrm{s}$ ratio. Its advantage is the strong emission of both involved lines. Also this $\mathrm{t} / \mathrm{t}$ ratio shows a general $n_{\mathrm{e}}$ dependence, the overall value of the measured ratio does not match the predictions of the CRMs which are shown in Figure 11. The predictions without high Ry states deliver significantly higher numbers of the ratio, which means that the population of the $3^{3} \mathrm{D}$ state is overestimated in respect to the $3^{3} \mathrm{~S}$ state. The discrepancy between the models becomes higher with rising density. Also the separation of the lines with the same number for the ratio grows with rising temperature. This means that the ratio becomes less sensitive to $n_{\mathrm{e}}$ changes. Reversed this means that small changes of the measured ratio now yield higher changes of the calculated density. Nevertheless, the overall measured values of the ratio (cf. Table A1) are smaller than the predictions, which lead to significantly too low electron densities (cf. Figure 17).

To achieve a strong temperature dependence, ratios between singlet and triplet lines are used, with the classical combination being the 706/728 (t/s) ratio. This general $T_{\mathrm{e}}$ dependence leads in most of the $(\mathrm{s} / \mathrm{t})$ ratios to a strong change of their value along the plasma radius as shown in Figure 9 (left). As an example for a clearly separated $T_{\mathrm{e}}$ dependence, the 501/587 (s/t) ratio is plotted in Figure 12 as a comparison of the three CRMs. Again the discrepancy between the ADAS code and the hybrid code with Ry is the smallest, although the differences are more prominent than in the comparison of ratios from the same species. Together with the absolute value of the measured ratio from Table A1 and Figure 14 which lies in the range of 0.01-0.11, one can see that in this case the hybrid code without high Ry is best suitable to describe the data (cf. Figure 15) as separatrix temperatures between 30 and $50 \mathrm{eV}$ for L-modes and $100 \mathrm{eV}$ for the H-mode are assumed [37].

The discrepancy of the results in respect to the number of considered Rydberg 

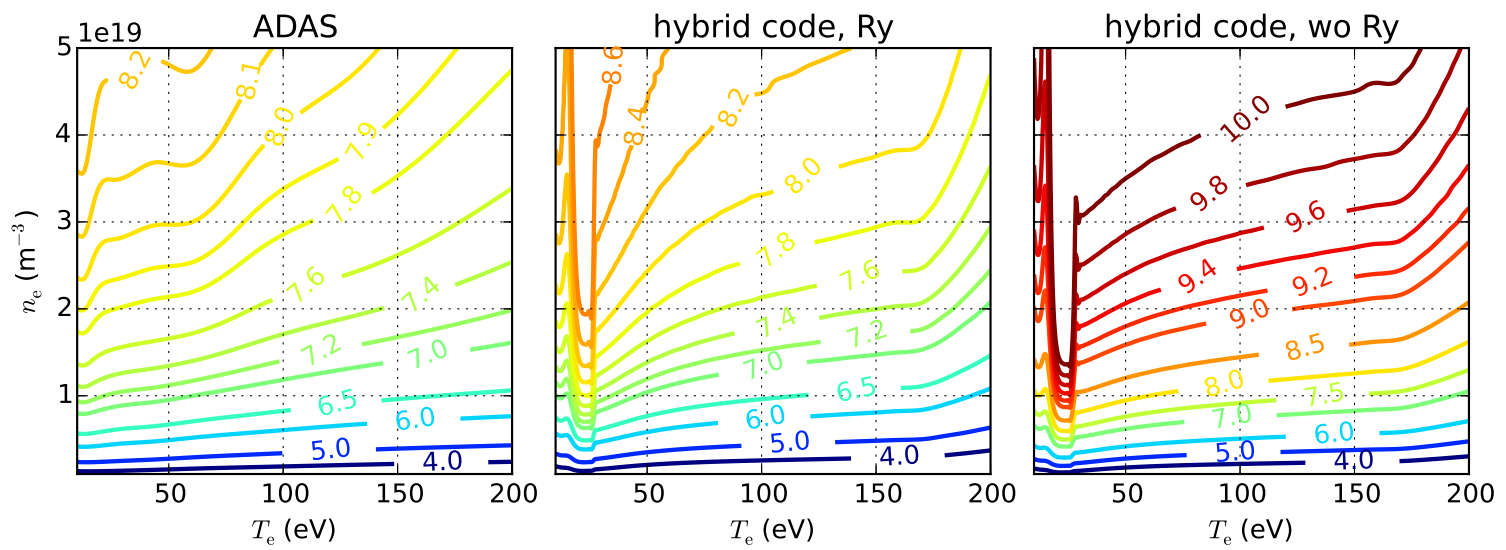

Figure 11: Comparison of the $T_{\mathrm{e}}$ and $n_{\mathrm{e}}$ behaviour of the $587 / 706(\mathrm{t} / \mathrm{t})$ ratio for three different CRMs.
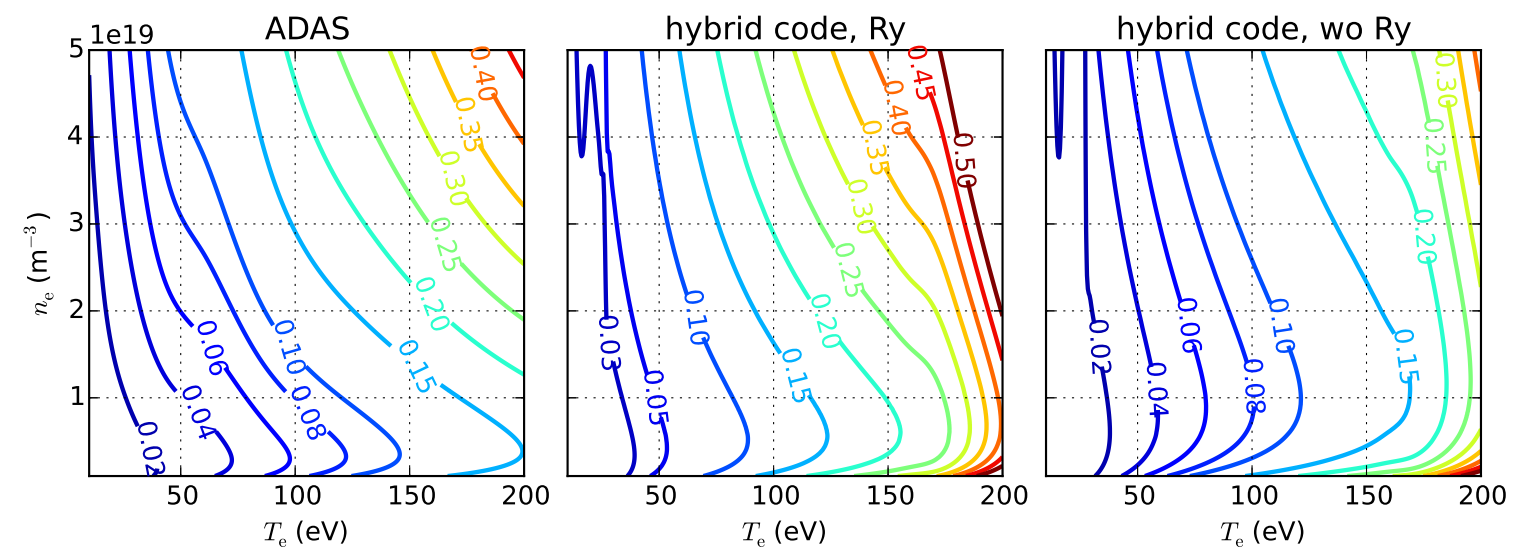

Figure 12: Comparison of the $T_{\mathrm{e}}$ and $n_{\mathrm{e}}$ behaviour of the $501 / 587(\mathrm{~s} / \mathrm{t})$ ratio for three different CRMs.

states strongly depends on the $n_{\mathrm{e}}$ and $T_{\mathrm{e}}$ range as well as on the chosen ratio. A direct comparison of the photon emission coefficient of all states shows that especially all lines starting from an $n=4$ level are highly affected by the treatment of the Rydberg states. On the one hand, the photo emission coefficient and thus the population density of all $n=4$ states is overestimated for the hybrid code without high Rydberg states with respect to the ADAS results. On the other hand the inclusion of states up to $n=500$ leads to a lower population density compared to ADAS. From this general result one cannot directly conclude which model describes the physics best. In fact one has to assume that dependent on the electron density different treatments of the Rydberg states may be necessary, especially because highly excited Rydberg states in the high $n_{\mathrm{e}}$ range may be physically impossible [25].

Beside the $n=4$ states also the other states are affected by the Rydberg state treatment (cf. Figure 11). Mostly the population density of all states is overestimated without the high Rydberg states, also the amount depends strongly on the specific state and the $n_{\mathrm{e}}$ 
and $T_{\mathrm{e}}$ region. This can be seen in Figure 10 and Figure 11 where the results are closest for small $n_{\mathrm{e}}$ values.

In addition to the treatment of high Rydberg states it can be seen that the predicted emission intensities within one model are not consistent for different line ratios. This is shown in section 6, Figure 17 where several ratios are used to calculate $n_{\mathrm{e}}$ and $T_{\mathrm{e}}$ profiles. Especially problematic is the temperature evaluation where ratios between singlet and triplet lines are formed and thus the singlet-triplet mixture is from highest importance. Several parameters as the initial energy of the helium atoms or the species dependent ionisation or charge-exchange (CX) losses may affect the s/t mixture. Whereas [38] shows that helium-deuteron CX losses can be neglected compared to electron-impact ionisation, the species dependent rate coefficients for $\mathrm{CX}$ between $\mathrm{He}^{+}$and hydrogen isotopes (cf. [39]) may contribute as additional population channel which favours triplet state population. This mostly would lead to higher temperatures evaluated from $\mathrm{s} / \mathrm{t}$ ratios in regions where neutral hydrogen is present.

As one of the best suitable examples for line ratio pairs, the contour plot in Figure 13 shows the values of the $501 / 504(\mathrm{~s} / \mathrm{s}) n_{\mathrm{e}}$ and the $501 / 587(\mathrm{~s} / \mathrm{t}) T_{\mathrm{e}}$ sensitive ratio, exemplarily as result of the static CRM based on ADAS data and routines [34].

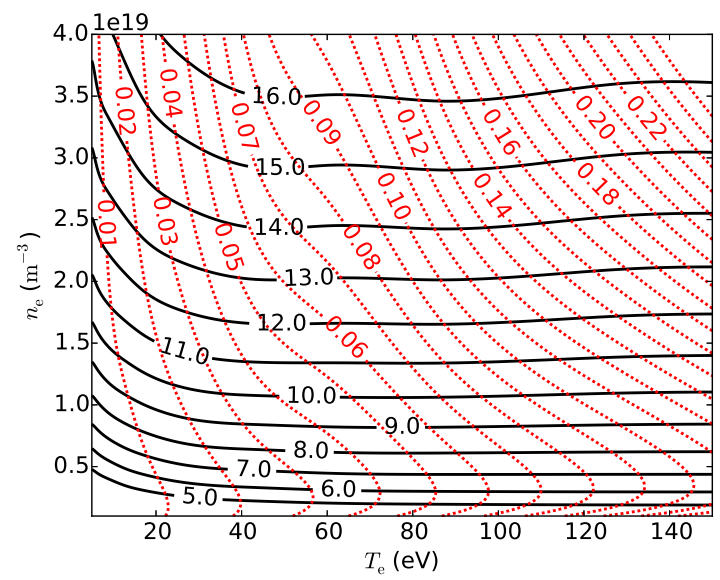

Figure 13: Temperature and density dependence of the s/t line ratio 501/587 (red, dashed) and the s/s line ratio 501/504 (black, solid). The data are based on the ADAS data set 'pec96\#he_pju\#he0.dat' [34].

Together with the measured line ratios in Figure 14, this grid can be used in a static approach, assuming a local equilibrium, to translate the measured ratio into temperature and density values as done in the next section where also the time dependent approach $[25]$ is discussed. 


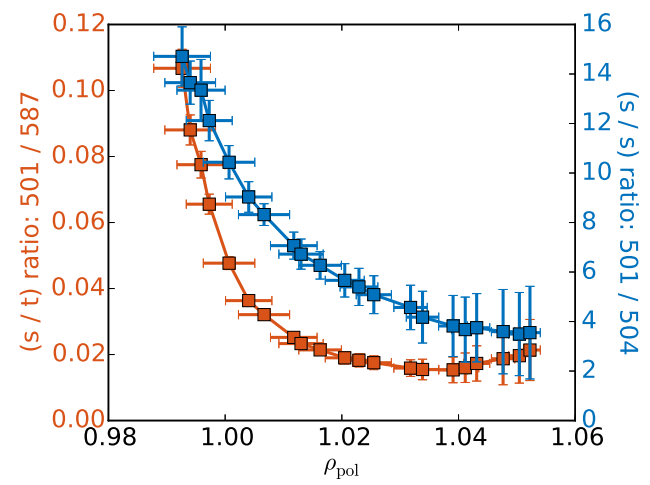

Figure 14: Radial profile of the $501 / 504(\mathrm{~s} / \mathrm{s}) n_{\mathrm{e}}$ and the $501 / 587(\mathrm{~s} / \mathrm{t}) T_{\mathrm{e}}$ sensitive ratio. Data from AUG discharges \#32 030 and \#32 031 for L-mode, in the time span of $2.14-2.37 \mathrm{~s}$.

\section{Comparison of $n_{\mathrm{e}}$ and $T_{\mathrm{e}}$ profiles}

In the simplest approach for profile evaluation, Figure 13 can be used as a look up table to translate the measured value pairs of the ratios from Figure 14 in a static way into $n_{\mathrm{e}}$ and $T_{\mathrm{e}}$ data. In some cases this method is limited by the relatively long relaxation times of the triplet ${ }^{3} \mathrm{~S}$ metastable term of helium which populates the triplet spin system. To handle this effect, a time dependent analytical solution, called the hybrid code (cf. [25]), was developed which models the time dependent population density of the two spin systems. In contrast to the static approach, this method takes care of the spatial distance between the measurement points. By adding the helium propagation velocity, the code considers the history along the helium trajectory for the proper distribution into single and triplet states. This mainly affects the electron temperature sensitive line ratios.

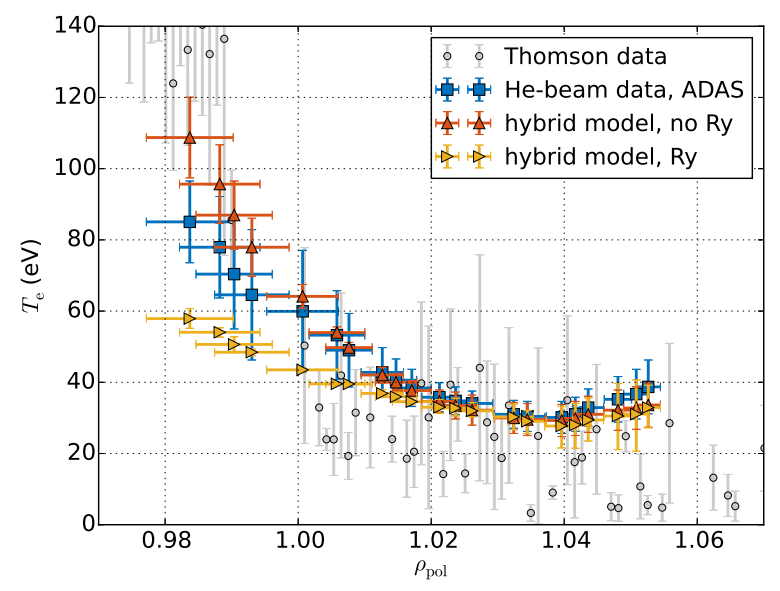

Figure 15: L-mode temperature profile in AUG discharge \#32030, $t=2.14-2.37 \mathrm{~s}$. Compared are the results from the 501/587 ratio calculated with three CRMs. The hybrid model with and without high Ry and the ADAS CRM. 
Looking at the $T_{\mathrm{e}}$ sensitive line ratio in Figure 14 one can see that the ratio rises again for higher radii, caused by the long relaxation times for small electron densities. Translating this measured ratio in a static way into electron temperature values as done in Figure 15 with the ADAS data set, leads to a temperature profile which rises also again at higher radii. This nonphysical effect can be mostly compensated by applying the hybrid code, as also shown in [25].
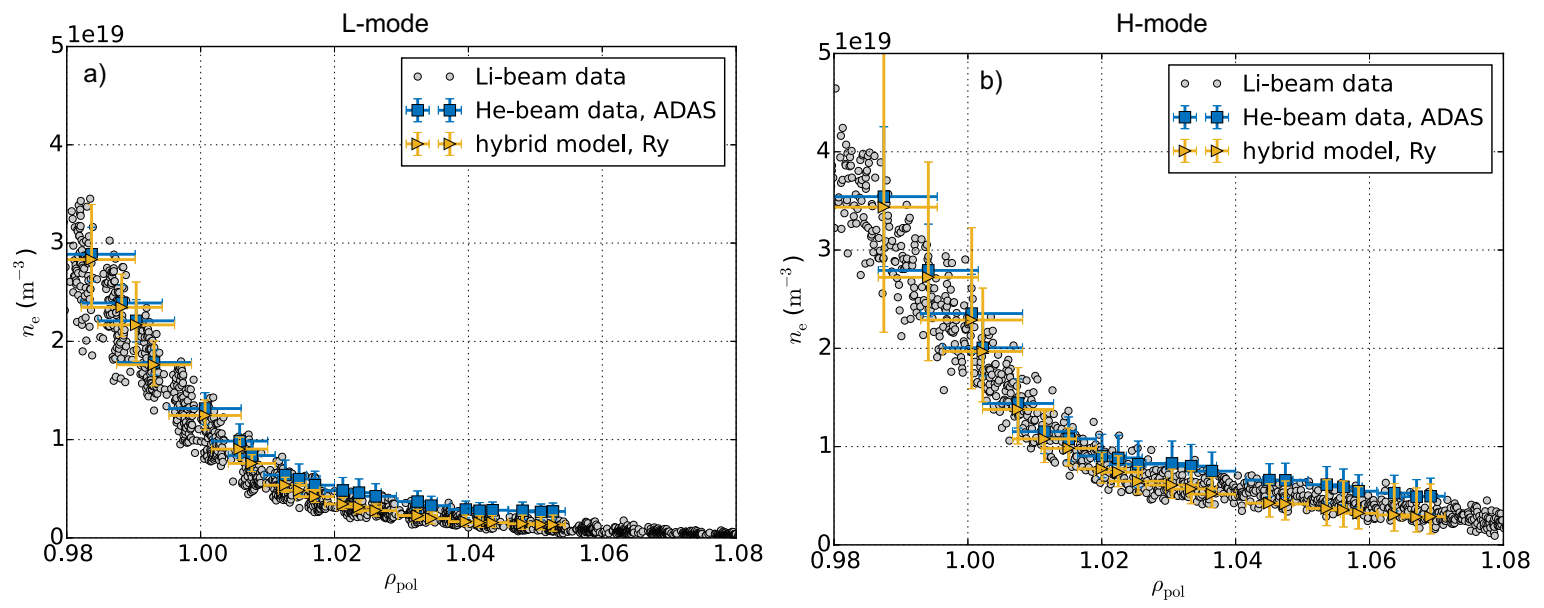

Figure 16: Density profile in AUG discharge \#32030, for a) the L-mode at $t=$ $2.14-2.37 \mathrm{~s}$, and $\mathrm{b}$ ) the H-mode at $t=3.34-3.57 \mathrm{~s}$. The reconstructed profiles out of the 501/504 ratio with the hybrid model with Ry and the ADAS code are compared to the Li-beam data.

For the electron density, the hybrid code delivers comparable results to the ADAS model in both, L- and H-mode cases (cf. Figure 16). The results are in both cases in excellent agreement with the Li-beam data as reference. Nevertheless, this good agreement is only achieved with the 504/667 and the 501/504 ratios as plotted in Figure 17. The remaining three ratios with a general $n_{\mathrm{e}}$ dependence deliver significantly too low or too high values. This shows that different ratios are not described in a self consistent way, also the models deliver consistent results within one chosen ratio for the density.

For electron temperature sensitive line ratios, the models also coincide for one fixed ratio within the error bars and under consideration of the time dependent effects in the far SOL for small $n_{\mathrm{e}}$ and $T_{\mathrm{e}}$ values, as shown in Figure 15. In regions of high temperate and density however, a significant discrepancy between the models is found. This discrepancy arises from the different number and treatment of Rydberg states in the models. To handle this effect, a revised handling of the Rydberg states is necessary as discussed in section 5. Compared to the data of the Thomson scattering diagnostic, the model without the high Ry states delivers the best results in the high temperature region. 


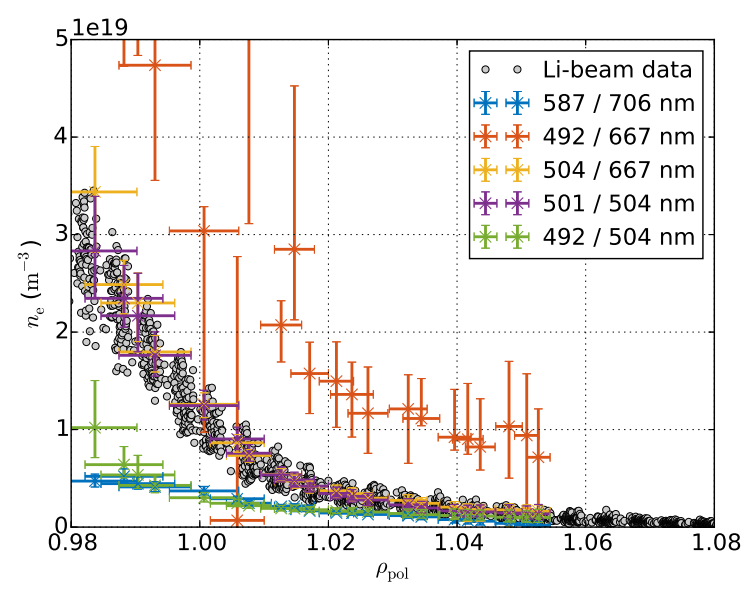

Figure 17: L-mode density profile at AUG. Compared are the results from the hybrid model with Ry form several ratios with general $n_{\mathrm{e}}$ dependence (cf. Figure 9, right, marked with asterisks). The used $T_{\mathrm{e}}$ ratio for the self consistent analysis is always the $501 / 587$ ratio.

\section{Summary and conclusion}

A thermal helium beam diagnostic based on a piezo valve and a spectrometer setup was implemented to the ASDEX Upgrade tokamak. This enabled a comprehensive measurement of spatially resolved He I emission lines in the edge region of a fusion plasma. The aim of this study is the selection of suitable ratios in combination with a proper collisional-radiative model $(\mathrm{CRM})$ to describe electron density and temperature profiles. Seven measured lines and thus 21 different ratios allowed a comparison of different CRMs and their underlying processes and assumptions. It is shown that the state of art CRMs are not able to describe all measured lines in a consistent way and that the calculated temperature and density profiles strongly depend on the chosen ratio. Especially a better treatment of highly excited Rydberg states as well as the additional charge-exchange process of $\mathrm{He}^{+}$and neutral hydrogen might be necessary to achieve more consistent results. The shown comparison based on experimental values is an excellent basis for the improvement of the CRM. Therefore these additional processes will be analysed and implemented in the next version of the hybrid code.

Consistently within the existing CRMs, especially the 504/667 and the 501/504 (s/s) ratios are best suitable for electron density evaluation with an excellent agreement to the lithium beam diagnostic at AUG. For the electron temperature the 501/587 (s/t) ratio is useful although the discrepancy between the CRMs is not fully resolved.

Nevertheless is has been shown that the brilliant signal level will allow faster measurements in the future without increasing the amount of injected helium. The small amount of necessary diagnostic gas does not lead to a measurable disturbance of the plasma. This will allow continuous and simultaneous measurements of the electron temperature and density to resolve filaments in the plasma edge region with a high 
spacial and temporal resolution.

Therefore and for the ongoing use of the helium line ratio technique as a standard diagnostic at ASDEX Upgrade, several hardware improvements were undertaken. A new optical head improves the spatial resolution up to $3 \mathrm{~mm}$. A newly designed multichromator system allows the simultaneous measurement of four wavelength with 32 channels and a temporal resolution up to $900 \mathrm{kHz}$. This should enable the diagnostic system to study turbulent transport in the edge region of AUG.

\section{Acknowledgments}

This work has been carried out within the framework of the EUROfusion Consortium and has received funding from the Euratom research and training programme 20142018 under grant agreement No 633053. The views and opinions expressed herein do not necessarily reflect those of the European Commission.

This work was funded in part by the U.S. Department of Energy under Grant DESC00013911 and DE-SC0014210.

The authors especially want to thank Dirk Wünderlich from IPP in Garching for helpful discussions about the helium CRM.

\section{Appendix A. Values for all measured line ratios.}


Table A1: Comparison of all measured line ratios for the first two time intervals in Figure 3. The maximum and minimum value of the ratios along the measured radius is given. The column with the value for $\max / \mathrm{min}$ shows the dependence of the given ratios for changes of $T_{\mathrm{e}}$ and $n_{\mathrm{e}}$ along the radius. The absolute emission intensity of the weaker line of a ratio limits the possible time resolution of measurements. Thus the relative intensity of the transitions with respect to the weakest line is given. The intensity is averaged along the radial profile for each time interval.

\begin{tabular}{clccccccccc}
\hline & & \multicolumn{3}{c}{ L-mode: } & $2.14-2.37 \mathrm{~s}$ & \multicolumn{3}{c}{ H-mode: $3.34-3.57 \mathrm{~s}$} \\
line & spin & max & min & $\max /$ & rel & \multicolumn{2}{c}{ max } & min & max/ & rel \\
ratio & type & ratio & ratio & min & int & ratio & ratio & min & int \\
\hline $447 / 492$ & $\mathrm{t} / \mathrm{s}$ & 5.24 & 1.61 & 3.25 & 7 & 4.84 & 1.22 & 3.98 & 8 \\
$447 / 501$ & $\mathrm{t} / \mathrm{s}$ & 6.64 & 1.03 & 6.47 & 9 & 6.59 & 0.65 & 10.1 & 10 \\
$447 / 504$ & $\mathrm{t} / \mathrm{s}$ & 24.1 & 15.2 & 1.59 & 1 & 34.1 & 10.3 & 3.31 & 1 \\
$447 / 587$ & $\mathrm{t} / \mathrm{t}$ & 0.14 & 0.06 & 2.20 & 21 & 0.09 & 0.06 & 1.50 & 25 \\
$447 / 667$ & $\mathrm{t} / \mathrm{s}$ & 0.87 & 0.16 & 5.51 & 21 & 0.81 & 0.10 & 7.96 & 25 \\
$447 / 706$ & $\mathrm{t} / \mathrm{t}$ & 0.58 & 0.26 & 2.23 & 21 & 0.46 & 0.28 & 1.63 & 25 \\
$492 / 501$ & $\mathrm{~s} / \mathrm{s}$ & 1.31 & 0.62 & 2.11 & 7 & 1.36 & 0.53 & 2.55 & 8 \\
$492 / 504$ & $\mathrm{~s} / \mathrm{s}$ & 9.42 & 4.55 & 2.07 & 1 & 8.49 & 6.82 & 1.24 & 1 \\
$492 / 587$ & $\mathrm{~s} / \mathrm{t}$ & 0.07 & 0.02 & 3.57 & 7 & 0.05 & 0.02 & 2.71 & 8 \\
$492 / 667$ & $\mathrm{~s} / \mathrm{s}$ & 0.17 & 0.10 & 1.70 & 7 & 0.17 & 0.08 & 2.02 & 8 \\
$492 / 706$ & $\mathrm{~s} / \mathrm{t}$ & 0.36 & 0.06 & 6.25 & 7 & 0.38 & 0.06 & 6.47 & 8 \\
$501 / 504$ & $\mathrm{~s} / \mathrm{s}$ & 14.8 & 3.62 & 4.09 & 1 & 15.9 & 5.18 & 3.07 & 1 \\
$501 / 587$ & $\mathrm{~s} / \mathrm{t}$ & 0.11 & 0.02 & 6.79 & 9 & 0.10 & 0.01 & 6.83 & 10 \\
$501 / 667$ & $\mathrm{~s} / \mathrm{s}$ & 0.16 & 0.12 & 1.35 & 9 & 0.16 & 0.12 & 1.29 & 10 \\
$501 / 706$ & $\mathrm{~s} / \mathrm{t}$ & 0.56 & 0.05 & 12.3 & 9 & 0.71 & 0.04 & 16.5 & 10 \\
$504 / 587$ & $\mathrm{~s} / \mathrm{t}$ & 0.01 & 0.00 & 2.15 & 1 & 0.01 & 0.00 & 2.23 & 1 \\
$504 / 667$ & $\mathrm{~s} / \mathrm{s}$ & 0.04 & 0.01 & 3.47 & 1 & 0.02 & 0.01 & 2.45 & 1 \\
$504 / 706$ & $\mathrm{~s} / \mathrm{t}$ & 0.04 & 0.01 & 3.04 & 1 & 0.04 & 0.01 & 5.37 & 1 \\
$587 / 667$ & $\mathrm{t} / \mathrm{s}$ & 7.79 & 1.45 & 5.37 & 61 & 8.74 & 1.63 & 5.37 & 66 \\
$587 / 706$ & $\mathrm{t} / \mathrm{t}$ & 5.57 & 2.13 & 2.61 & 66 & 7.48 & 3.07 & 2.44 & 75 \\
$667 / 706$ & $\mathrm{~s} / \mathrm{t}$ & 3.64 & 0.35 & 10.4 & 61 & 4.54 & 0.35 & 12.9 & 66 \\
\hline & & & & & & & & & \\
\hline
\end{tabular}

\section{References}

[1] Willensdorfer M, Birkenmeier G, Fischer R, Laggner F M, Wolfrum E, Veres G, Aumayr F, Carralero D, Guimarãis L, Kurzan B and Team t A U 2014 Plasma Physics and Controlled Fusion 5625008 URL http://stacks.iop.org/0741-3335/56/i=2/a=025008

[2] Silva A, Manso M E, Cupido L, Albrecht M, Serra F, Varela P, Santos J, Vergamota S, Eusébio F, Fernandes J, Grossmann T, Kallenbach A, Kurzan B, Loureiro C, Meneses L, Nunes I, Silva F, Suttrop W and the ASDEX Upgrade Team 1996 Review of Scientific Instruments 67 4138-4145 URL http://dx.doi.org/10.1063/1.1147517

[3] Coor T, Cunningham S, Ellis R, Heald M and Kranz A 1958 Physics of Fluids 1 
411 ISSN 1070-6631 URL http://www-naweb.iaea.org/napc/physics/2ndgenconf/data/ Proceedings1958/papersVol32/Paper25\{_\}ol32.pdf

[4] Brosda B 1993 Modellierung von Helium-Atomstrahlen und ihr Einsatz zur Plasmadiagnostik der Tokamakrandschicht Ph.D. thesis Ruhr-Universität Bochum

[5] Schweer B, Mank G and Pospieszczyk A 1992 Journal of nuclear ... 198 174-178 URL http: //www.sciencedirect.com/science/article/pii/S0022311506800267

[6] Ahn J W, Craig D, Fiksel G, Den Hartog D J, Anderson J K and O'Mullane M G 2006 Development of fast helium beam emission spectroscopy on MST Review of Scientific Instruments vol 77 ISSN 00346748

[7] Kajita S, Ohno N, Takamura S and Nakano T 2006 Physics of Plasmas 13 1-9 ISSN 1070664X

[8] Sieglin B 2010 Measurement of the Electron Temperature in the Scrape-Off Layer of ASDEX Upgrade by Line Integrated Helium I Emission Lines Diploma thesis TUM

[9] Boivin R F, Kline J L and Scime E E 2001 Physics of Plasmas 8 5303-5314 ISSN 1070664X

[10] Ma S, Howard J, Blackwell B D and Thapar N 2012 Review of Scientific Instruments 83033102 ISSN 00346748 URL http://www.ncbi.nlm.nih.gov/pubmed/22462901

[11] Lashkul S I, Altukhov a B, D'yachenko V V, Esipov L a, Kantor M Y, Kuprienko D V, Lebedev a D, Nikerman Y a and Popov a Y 2012 Plasma Physics Reports 38 851-862 ISSN 1063-780X URL http://link.springer.com/10.1134/S1063780X12110062

[12] Namba S, Andruczyk D, Takiyama K, Ueno D, Furukawa S and James B W 2006 Japanese Journal of Applied Physics, Part 1: Regular Papers and Short Notes and Review Papers 45 8099-8103 ISSN 00214922

[13] Brix M 1998 Messung von Elektronentemperatur und -dichte mittels Heliumstrahldiagnostik im Randschichtplasma eines Tokamaks Phd thesis Ruhr-Universität Bochum

[14] Tabares F L, Tafalla D, Herrero V and Tanarro I 1997 Journal of Nuclear Materials 241-243 1228-1233 ISSN 00223115

[15] Schmitz O, Beigman I L, Vainshtein L A, Schweer B, Kantor M, Pospieszczyk A, Xu Y, Krychowiak M, Lehnen M, Samm U and Unterberg E A 2008 Plasma Physics and Controlled Fusion 50 115004 ISSN 0741-3335 URL http://stacks.iop.org/0741-3335/50/i=11/a=115004?key= crossref .59869d677a4e0d5c8dba1c0a7480fd7e

[16] Davies S, Morgan P, Ul'Haq Y, Maggi C, Erents S, Fundamenski W, Horton L, Loarte A, Matthews G, Monk R and Stangeby P 1997 Parallel electron temperature and density gradients measured in the JET MkI divertor using thermal helium beams

[17] Carraro L, Pol G D, Puiatti M E, Sattin F, Scarin P and Valisa M 1999 Plasma Physics and Controlled Fusion 42 1-14 ISSN 0741-3335

[18] Agostini M, Scarin P, Cavazzana R, Fassina A, Alfier A and Cervaro V 2010 Optical measurements for turbulence characterization in RFX-mod edge Review of Scientific Instruments vol 81 ISBN 0034-6748 ISSN 00346748

[19] Agostini M, Scarin P, Cavazzana R, Carraro L, Grando L, Taliercio C, Franchin L and Tiso A 2015 Review of Scientific Instruments 86123513 ISSN 0034-6748 URL http://scitation. aip.org/ content/aip/journal/rsi/86/12/10.1063/1.4939003

[20] Barbui T, Krychowiak M, König R, Schmitz O, Muñoz Burgos J M, Schweer B, Terra A and Team W X 2016 Review of Scientific Instruments 87 URL http://scitation.aip.org/content/ aip/journal/rsi/87/11/10.1063/1.4962989

[21] Jones O M, Meyer O, Xu H, Sorrentino S, Baude R, Escarguel A, Harris J H and Rd S $201643 r d$ EPS Conference on Plasma Physics 1-4

[22] Pospieszczyk A, Chevalier G, Hirooka Y, Conn R W, Doerner R and Schmitz L 1992 Nuclear Inst. and Methods in Physics Research, B 72 207-223 ISSN 0168583X

[23] Ray H B, Biewer T M, Fehling D T, Isler R C and Unterberg E A 2016 Review of Scientific Instruments 87 11E711 ISSN 0034-6748 URL http://scitation.aip.org/content/aip/ journal/rsi/87/11/10.1063/1.4959796

[24] Muñoz Burgos J M, Barbui T, Schmitz O, Stutman D and Tritz K 2016 Review of Scientific 
Instruments 87 11E502 ISSN 0034-6748 URL http://scitation.aip.org/content/aip/ journal/rsi/87/11/10.1063/1.4955286

[25] Muñoz Burgos J M, Schmitz O, Loch S D and Ballance C P 2012 Physics of Plasmas 19 012501 ISSN 1070664X URL http://scitation.aip.org/content/aip/journal/pop/19/1/ 10.1063/1.3672230

[26] Griener M, Schmitz O, Bald K, Bösser D, Cavedon M, Marné P D, Eich T, Fuchert G, Herrmann A, Kappatou A, Lunt T, Rohde V, Schweer B, Sochor M, Stroth U, Terra A and Wolfrum E 2017 Review of Scientific Instruments 8833509 URL http://dx.doi.org/10.1063/1.4978629

[27] Kruezi U 2006 Entwicklung einer Heliumstrahldiagnostik zur Messung der Elektronendichte und -temperatur mit hoher räumlicher und zeitlicher Auflösung Dissertation Heinrich-HeineUniversität Düsseldorf

[28] Kramida A, Ralchenko Y and Reader J 2015 NIST Atomic Spectra Database (ver. 5.3) Online, 2016, September 12, National Institute of Standards and Technology, Gaithersburg, MD. URL http://physics.nist.gov/asd

[29] De La Cal E 1998 Nuclear Instruments and Methods in Physics Research, Section A: Accelerators, Spectrometers, Detectors and Associated Equipment 403 490-498 ISSN 01689002

[30] Viezzer E, Pütterich T, Dux R and McDermott R 2012 Review of Scientific Instruments 83103501 ISSN 1089-7623 URL http://www.ncbi.nlm.nih.gov/pubmed/23130787

[31] Cavedon M 2016 Technische Universität München Max-Planck-Institut für Plasmaphysik The role of the radial electric field in the development of the edge transport barrier in the ASDEX Upgrade tokamak Ph.D. thesis

[32] Cavedon M, Pütterich T, Viezzer E, Dux R, Geiger B, McDermott R M, Meyer H and Stroth U 2017 Review of Scientific Instruments 8843103 URL http://dx.doi.org/10.1063/1.4979801

[33] Sun H J, Wolfrum E, Eich T, Kurzan B, Potzel S, Stroth U and the ASDEX Upgrade Team 2015 Plasma Physics and Controlled Fusion 57125011 URL http://stacks.iop.org/0741-3335/ $57 / i=12 / a=125011$

[34] Summers H P 2004 The ADAS User Manual, version 2.6, http://www.adas.ac.uk URL http: //www.adas.ac.uk

[35] Summers H P, Dickson W J, O'Mullane M G, Badnell N R, Whiteford A D, Brooks D H, Lang J, Loch S D and Griffin D C 2006 Plasma Physics and Controlled Fusion 48 263293 ISSN 0741-3335 URL http://stacks . iop. org/0741-3335/48/i=2/a=007?key=crossref . f88c8fb59bc0bbcf9fc671f91cd4d8bd

[36] Munoz Burgos J M, Loch S D, Ballance C P and Boivin R F 2009 Astronomy and Astrophysics 500 1253-1261 ISSN 0004-6361 URL http://www. aanda.org/10.1051/0004-6361/200911743

[37] Sun H J, Wolfrum E, Kurzan B, Eich T, Lackner K, Kardaun O, Faitsch M, Potzel S, Stroth U and Team t A U 2017 submitted to: Plasma Physics and Controlled Fusion

[38] Muñoz Burgos J M, Agostini M, Scarin P, Stotler D P, Unterberg E A, Loch S D, Schmitz O, Tritz K and Stutman D 2016 Physics of Plasmas 23053302 ISSN 1070-664X URL http: //scitation.aip.org/content/aip/journal/pop/23/5/10.1063/1.4948554

[39] Loreau J, Ryabchenko S, Dalgarno A and Vaeck N 2011 Phys. Rev. A 8452720 URL http: //link.aps.org/doi/10.1103/PhysRevA.84.052720 